\title{
EVALUATION OF THE PHARMACY SUPPORT SYSTEM IN THE DETECTION OF DRUG-RELATED PROBLEMS
}

\author{
TRI MURTI ANDAYANI ${ }^{1 *}$, FITA RAHMAWATI ${ }^{1}$, RIFKY ROCHMAN ${ }^{2}$, ARRISA DWI NINGRUM ${ }^{3}$
}

${ }^{1}$ Department of Pharmacology and Clinical Pharmacy, Faculty of Pharmacy, Universitas Gadjah Mada, Indonesia. ${ }^{2}$ Department of Pharmaceutics, Faculty of Pharmacy, Universitas Gadjah Mada, Indonesia. ${ }^{3}$ Department of Pharmacy, Faculty of Pharmacy, Universitas Gadjah Mada, Indonesia. Email: trimurtia@yahoo.com

Received: 18 October 2016, Revised and Accepted: 24 January 2017

ABSTRACT

Objective: The efficiency and speed of a system in identifying drug-related problems (DRPs) can support and optimize the performance of pharmacists. The objective of this research was to determine the time of analysis and the number of DRPs' incidences identified by pharmacists with and without pharmacy support systems (PSS).

Methods: The present research was observational with cross-sectional design. The data collection was done prospectively in outpatients at hospital during January 2016. Observations were conducted to the difference between the analysis and the number of DRPs' incidence identified by the pharmacists with and without the assistance of PSS. The research population was outpatient at a number of hospitals in Yogyakarta. The tools used in this research were PSS which were a clinical information system that can identify potential DRPs and included three main parts, namely, the patients' profile, drug information, and analysis of DRPs. The identification result of DRPs was consisted of six DRPs categories, i.e., without treatment indication, treatment without indication, ineffective drug, too low dose, too high dose, and undesirable drug reactions.

Results: Pharmacists without PSS require faster time to analyze the prescription of outpatients. The time majority spent by pharmacists with PSS in reviewing the patients' prescription lies in the length of time the patients enter the therapy-related data obtained manually by patients until the warning being displayed and making clinical decision related to the DRPs. The statistical test result using Goodness of fit test and Fisher between categories of DRPs incidences detected by pharmacists with and without the assistance of PPS indicates significant differences (p<0.05). Pharmacists with PSS can detect DRPs that are not detected by the pharmacist without PSS. DRPs mostly identified by pharmacists with the assistance of PSS are drug interactions and improper doses. Improper doses primarily identified in geriatric and pediatric patients' prescription.

Conclusion: Pharmacists with PSS software can detect DRPs that are not detected by pharmacists only. However, pharmacists using the software PSS require a longer time in the identification of DRPs compared to pharmacists without using PSS.

Keywords: Pharmacy support systems, Drug-related problems, Outpatients.

(C) 2017 The Authors. Published by Innovare Academic Sciences Pvt Ltd. This is an open access article under the CC BY license (http://creativecommons. org/licenses/by/4. 0/) DOI: http://dx.doi.org/10.22159/ajpcr.2017.v10s2.19497

\section{INTRODUCTION}

Drug-related problems (DRPs) are events or circumstances related to drug therapy, which potentially or actually affect clinical outcomes of the patients. DRPs are capable of resulting significant morbidity and mortality and contribute to soaring health costs [1]. Several strategies have been done to prevent drug-related problems, for instance, the evaluation of drug treatment, education of health-care workers or patients, assessing treatment and participation of clinical pharmacists in health professional team. Daily activities in hospital characterized by a high number of patients, very limited time, and speed of services provide unlimited space for evaluating drug therapy [2]. Professional skills of pharmacists in identifying the DRPs cover knowledge not only regarding drug but also evidence-based guidelines and contemporary management of various medical conditions also play a role in the incidence of DRPs. Thereby, that the information technology in treatment management process has been suggested to improve appropriate and safe treatment. The utilization of computerized-based information technology is proven to efficiently assist the pharmacists in identifying potential DRPs [3-6].

Study center and consultation: Industry, management, and pharmacy services of Gadjah Mada University have developed software of clinical information system called Gama pharmacy support system (GPSS), in which this system is able to assess prescriptions in outpatient and provide warning on DRPs potential. GPSS is comprised three main parts, i.e., the patients' profile, drug information, and DRPs analysis. The presence of such system is expected to assist pharmacists in making clinical decisions associated with patients' treatment and to prevent DRPs incidences.

In the medical technology or intervention, a diagnostic test must be evaluated before being introduced into daily practice. Diagnostic accuracy plays a pivotal role in clinical evaluation [7]. The accuracy of a diagnostic test depends on the sensitivity and specificity. The computation result of GPSS diagnostic value has shown the sensitivity value of $97.2 \%$, specificity of $45.2 \%$, positive predictive value of $66.7 \%$, and negative predictive value of $93.4 \%$. The sensitivity value of $97.2 \%$ suggests that pharmacists with the assistance of GPSS are able to detect $95 \%$ of patients at risk of potential DRPs. The specificity value of $45.2 \%$ shows the capability of pharmacists with the help of GPSS to determine $45.2 \%$ of patients not at risk of potential DRPs. Previous research suggests that the safety warning system against drug use should have high sensitivity and specificity, provide clear information, not inhibit the workflow, and facilitate the handling upon warnings in safe and efficient manner [8]. However, there is no practical system that is able to achieve the sensitivity and specificity up to $100 \%$ [9]. The current system tends to have high sensitivity but generally low in specificity [10].

The efficiency and speed of a system in identifying DRPs can support and optimize the performance of pharmacists. Hatfield et al. (2014) reports that the implementation of computerized provider order entry system can allocate more time on clinical activities to strengthen clinical 
services through monitoring in therapeutic regimens. The objective of this research was to determine the time of analysis and the number of DRPs incidences identified by pharmacists with and without GPSS assistance [11].

\section{METHODS}

The present research was observational with cross-sectional design. The data collection was done prospectively in outpatients at hospital during January 2016. Observations were conducted to the difference between the analysis and the number of DRPs incidence identified by the pharmacists with and without the assistance of GPSS.

The research population was patients attending outpatient treatment at a number of hospitals in Yogyakarta with patient inclusion criteria of pediatric, geriatric, and heart disorders. The patients that had met inclusion criteria are identified with the presence or absence of potential DRPs. Furthermore, measurement was undertaken for analysis time and total DRPs incidences.

The tools used in this research were GPSS which were a clinical information system that can identify potential DRPs and included three main parts, namely, the patients' profile, drug information, and analysis of DRPs. The information data were consisted of drug's name, indication, mechanism of actions, doses, drug interactions, side effects, and contraindications. The references employed for database creation of GPSS were Drug Information Handbook, National Formulary, Drug Interaction Facts, MIMS, and Beers Criteria (2012).

Drug analysis results were displayed in the form of warning, i.e., too big or too small; too frequent or less for the frequency; improper drug use in geriatric patients (Beers criteria); and drug interactions. The identification result of DRPs was classified according to Cipolle et al. (2004), consisted of six DRPs categories, i.e. without treatment indication, treatment without indication, ineffective drug, too low dose, too high dose, and undesirable drug reactions [12].

To determine whether differences existed in analysis time of DRPs by pharmacists with and without the assistance of GPSS, Mann-Whitney test was employed. Statistical test was done to determine whether there were significant differences in DRPs incidence number, and goodness of fit test was used. The statistical analysis was undertaken with a confidence level of 95\%. Ethical Clearance was obtained from the medical faculty of Universitas Gadjah Mada Yogyakarta, Indonesia.

\section{RESULTS AND DISCUSSION}

In the development of health information technology, the validity and evaluation tests should be done beforehand to determine the ability of such system. GPSS is an electronic system supporting pharmaceutical clinical decision to detect potential DRPs which becomes one of the solutions to reduce, prevent, and resolve DRPs (Table 1).

GPSS validity tests has been carried out in pediatric, geriatric, and cardiovascular disorders. The results showed that the sensitivity of GPSS was $97.2 \%$ and specificity was $45.2 \%$. Furthermore, an evaluation was done in this research on the analysis time and the type of DRPs detected by GPSS.

On any computer system, speed is a very crucial parameter for users' acceptance in such system. Recommendation should appear exactly at the time of decision-making. When the application speed is slow, the users' satisfaction decreases. Bates et al. (2003) reported that speed of information system is the most appreciated parameter by users as it should be a priority [13].

Table 2 implies that there are differences in analysis time of DRPs by pharmacists with and without GPSS. The time majority spent by pharmacists with GPSS in reviewing the patients' recipe lies in the length of time the patients enter; the therapy-related data obtained
Table 1: Characteristic of the study subject

\begin{tabular}{ll}
\hline Characteristic & N (\%) \\
\hline Gender & \\
$\quad$ Male & $217(54.5)$ \\
Female & $181(45.5)$ \\
Age (years) & \\
$\quad<18$ & $198(49.7)$ \\
$18-60$ & $52(13.1)$ \\
$>60$ & $148(37.2)$ \\
Number of diagnose & \\
$<2$ & $239(60.1)$ \\
$>2$ & $159(39.9)$ \\
Number of drug prescribed & \\
$1-4$ & $266(66.8)$ \\
$>4$ & $132(31.2)$ \\
\hline
\end{tabular}

Table 2: Differences between time analysis conducted DRPs pharmacists and pharmacists with GPSS

\begin{tabular}{|c|c|c|c|}
\hline $\begin{array}{l}\text { Time analysis for DRPs } \\
\text { identification }\end{array}$ & $\mathbf{N}$ & $\begin{array}{l}\text { Median (second) } \\
\text { minimum-maximum }\end{array}$ & $\mathbf{p}$ \\
\hline Time analysis of PSS group & 300 & $110(13-300)$ & 0.000 \\
\hline $\begin{array}{l}\text { Time analysis of pharmacist } \\
\text { group }\end{array}$ & 300 & $24(4-196)$ & \\
\hline
\end{tabular}

GPSS: Gama pharmacy support system, PSS: Pharmacy support system,

DRPs: Drug-related problems

manually by patients until the warning being displayed and making clinical decision related to the DRPs. Hoeks (2014) states that when a computerized system requires the user to enter data manually, the process uses a quite long time, especially in outpatient services, where the time is critical.

Pharmacists without GPSS require faster time to analyze the prescription of outpatients. The high number of patients and the need for fast service result in limited time for pharmacists to review the prescriptions. Blix (2007) reports that activity in hospitals is characterized by high number of patients, limited time, and requires the speed of service, which results in limited space to the pharmacists to evaluate the therapy [2]. Hatfield et al. (2014) conducts a research by comparing the time required by the pharmacists to conduct distribution, administrative and clinical activities, and other activities (personal time) using computerized physician order entry (CPOE) and without CPOE, showing the presence of significant differences. The pharmacists' activities with $\mathrm{CPOE}$ require a longer time for clinical activity ( $>3$ minutes). The long period is used to strengthen clinical services through monitoring of therapeutic regimens [11].

The research conducted by Fok and Lo (2002) is to assess the pharmacists' workload in solving the problems related to recipes, including ambiguous, illegible, incomplete recipes, and to determine the problems and frequency of occurrence. Pharmacists use an average time of 20.1 minutes, starting from problem detection until the problems are resolved and the recipes are processed [14].

\section{The number and types of DRPs}

The difference of DRPs' types detected by pharmacists with and without the assistance of GPSS can be seen in Table 3.

The statistical test result using goodness of fit test and Fisher between categories of DRPs' incidences detected by pharmacists with and without the assistance of GPSS indicates significant differences $(p<0.05)$. Pharmacists with the help of GPSS can detect DRPs that are not detected by the pharmacist without the help of GPSS. The research results by Verdoorn et al. (2015) show that the use of clinical decision support system (CDSS) for treatment review can identify DRPs with 
Table 3: The difference of DRPs types detected by pharmacists with and without the assistance of GPSS

\begin{tabular}{lll}
\hline DRPs & N & \\
\cline { 2 - 3 } & Pharmacist+GPSS (\%) & Pharmacist (\%) \\
\hline Unnecessary drug needs additional drug therapy & $28(4.9)$ & $4(3.2)$ \\
Ineffective drug & $24(4.2)$ & 0 \\
Dosage too low and dosage too high drug interaction & $70(12.2)$ & $5(4.0)$ \\
& $232(40.6)$ & $14(11.3)$ \\
\hline
\end{tabular}

GPSS: Gama pharmacy support system, DRPs: Drug-related problems

Table 4: Incidence category of DRPs in pediatric, geriatric, and cardiovascular disease patient prescription

\begin{tabular}{|c|c|c|c|}
\hline \multirow[t]{2}{*}{ DRPs } & \multicolumn{3}{|l|}{$\mathbf{N}$} \\
\hline & Pediatric & Geriatric & Cardiovascular disease \\
\hline Unnecessary drug needs additional drug therapy & 0 & 5 & 15 \\
\hline Ineffective drug & 0 & 9 & 23 \\
\hline Dosage too low and dosage too high adverse drug reaction & 13 & 44 & 13 \\
\hline \multirow[t]{2}{*}{ Drug interaction } & 120 & 96 & 10 \\
\hline & 0 & 2 & 6 \\
\hline
\end{tabular}

DRPs: Drug-related problems

higher numbers, but the level of implementation is low. It can be seen from the DRPs that can be resolved before and after the use of CDSS having similar number.

DRPs mostly identified by pharmacists with the assistance of GPSS are drug interactions and improper doses. Improper doses primarily identified in geriatric and pediatric patients' prescription. The study by Tora et al. (2014) shows that the warning category for potential DRPs frequently identified by EES/CDSS in the age group >65 years old is drug-drug interaction with drug (38.6\%), geriatric warning (30.8\%), and duplications of therapy (25.6\%). The geriatric warning includes warning in drug inaccuracies in the elderly (11\% warning), extremely high doses (9\%), and more than 3 types of psychotropic drug (3\%) (Table 4) [4].

Drug interaction is warning generally appear at the most in patients with cardiovascular disorder. In moderate severity level, effects of between drug interactions have the potential to be life-threatening or capable of causing permanent damage. The largest drug interaction at moderate severity level is the interaction between bisoprolol and aspirin. The antihypertensive effects can be attenuated by aspirin through mechanisms of prostaglandin biosynthesis inhibition involved in activities hypertension activities. Meanwhile, the most common major interaction found is between clopidogrel and aspirin. The interaction effects can increase the risk of bleeding which can be life-threatening and may be higher in patients who are at high risk of transient ischemic attack or ischemic stroke [15]. Undesired effects because of drug interactions can be resolved by monitoring of clinic parameter, symptoms and signs of toxicity, doses adjustment, substituting drug with other alternative drugs, no longer administer one drug or preventing drug combination. The drug interactions appearing on GPSS are interaction mechanism, effects interaction, and interaction handling that could be used by pharmacists for the prevention of DRPs.

Incidence category of DRPs in geriatric and pediatric patient prescription at the most is improper doses. GPSS doses show a high sensitivity, but the specificity is rather low (79\%). GPSS tends to be too quick and issue warnings related to doses; thus, it must be reviewed by a pharmacist by viewing information on GPSS and judging the condition of patients. Isaksen et al. (1999) conducted a validation of computer-based program to identify patients at high risk of DRPs using six criteria regarding the use of drug, i.e., by drug administration $\geq 5$ drugs, $\geq 12$ doses per day, $\geq 4$ changes of therapeutic regimens, with $\geq$ chronic diseases, the history of noncompliance and drugs that require monitoring of drug level in the blood (therapeutic drug monitoring).
The criteria for the number of daily doses have the highest sensitivity of $91.7 \%$. It indicates that dose-related problems are highly sensitive to computerized analysis in the identification of DRPs in geriatric patients. Vulnerable populations, especially the pediatric and geriatric patients, are at risk of serious dosage errors, overdose in particular [16].

DRPs' categories are related to incorrect dosage identified by GPSS including the doses and excessive or less drug frequency, improper drug use because of improper drug dose as it does not correspond to the geriatric based on Beers criteria. The incorrect dosage category shows a specificity of $79 \%$ and a positive predictive value of $15 \%$. That is, GPSS is not able to detect geriatric patients actually have a dose-related DRPs. It is due to the PSS development system is only based on literature knowledge matched to the patients' drug profile without entering patients' profile data such as the general data of patients and clinical data of patients; thus, GPSS has not had the ability to search for daily dose recommended specifically for geriatric and pediatric. In addition, GPSS has not included guidelines for appropriate doses for patients with kidney disorders and drug doses should consider the laboratory data. Based on the few existing studies, GPSS development is still open to be conducted to determine the proper dose. Eppenga et al. (2012) conducted CDSS development including the determination of the drug dose that requires laboratory data and guidelines for the dose adjusted to kidney functions. The warning in this system includes interactions of drug-drug, drug-age, drug duplication, drug-disease, and dosage guidelines [17].

Based on the findings toward the review time of DRPs in prescription of outpatients treatment by the pharmacists with or without the support of GPSS, it shows that rapid analysis time does not guarantee the ability in detecting the presence of DRPs. The DRPs' analysis using GPSS requires a longer time due to prescription's data input process and requirement to read the warnings issued by GPSS. Warnings appearing in the GPSS should be revisited by the researchers as decision-makers in determining DRPs by means of reading the drug information found on GPSS and adapted to the patients' clinical condition. Computerbased access toward the drug completeness profile and warnings on potential problems recipe problems can reduce initial level of improper treatment [18].

\section{CONCLUSION}

GPSS can assist the pharmacists in identifying potential DRPs; nonetheless, the development of the tool is still required to enhance the specificity and sensitivity. 


\section{REFERENCES}

1. Johnson JA, Bootman JL. Drug-related morbidity and mortality and the economic impact of pharmaceutical care. Am J Health Syst Pharm 1997;54(5):554-8

2. Blix HS, Viktil KK, Reikvam A, Moger TA, Hjemaas BJ, Pretsch P, et al. The majority of hospitalised patients have drug-related problems: Results from a prospective study in general hospitals. Eur J Clin Pharmacol 2004;60(9):651-8.

3. Roten I, Marty S, Beney J. Electronic screening of medical records to detect inpatients at risk of drug-related problems. Pharm World Sci 2010;32(1):103-7.

4. Tora H, Bo H, Bodil L, Göran P, Birgit E. Potential drug related problems detected by electronic expert support system in patients with multi-dose drug dispensing. Int J Clin Pharm 2014;36(5):943-52.

5. Urbina O, Ferrández O, Luque S, Grau S, Mojal S, Pellicer R, et al. Patient risk factors for developing a drug-related problem in a cardiology ward. Ther Clin Risk Manag 2014;11:9-15.

6. Zaal RJ, Jansen MM, Duisenberg-van Essenberg M, Tijssen CC, Roukema JA, van den Bemt PM. Identification of drug-related problems by a clinical pharmacist in addition to computerized alerts. Int J Clin Pharm 2013;35(5):753-62.

7. Bossuyt PM, Reitsma JB, Linnet K, Moons KG. Beyond diagnostic accuracy: The clinical utility of diagnostic tests. Clin Chem 2012;58(12):1636-43.

8. van der Sijs H, Bouamar R, van Gelder T, Aarts J, Berg M, Vulto A. Functionality test for drug safety alerting in computerized physician order entry systems. Int J Med Inform 2010;79(4):243-51.

9. Coleman E, Aarts J, Kulikowski C. The dangerous decade. J Am Med
Inform Assoc 2012;19:2-5.

10. Weingart SN, Simchowitz B, Padolsky H, Isaac T, Seger AC, Massagli M, et al. An empirical model to estimate the potential impact of medication safety alerts on patient safety, health care utilization, and cost in ambulatory care. Arch Intern Med 2009;169(16):1465-73.

11. Hatfield MD, Cox R, Mhatre SK, Flowers WP, Sansgiry SS. Impact of computerized provider order entry on pharmacist productivity. Hosp Pharm 2014;49(5):458-65.

12. Cipolle RJ, Strand LM, dan Morley PC. Pharmaceutical Care Practice. New York: McGraw-Hill, Health Professions Division; 1998.

13. Bates DW, Kuperman GJ, Wang S, Gandhi T, Kittler A, Volk L, et al. Ten commandments for effective clinical decision support: Making the practice of evidence-based medicine a reality. J Am Med Inform Assoc 2003;10(6):523-30.

14. Fok M, Lo A. Quantifying the impact of prescription-related problems on pharmacy workload. Can J Hosp Pharm 2002;55:313-9.

15. Tatro DS. Drug Interaction Fact. St. Louis, MO: Facts and Comparisons; 2012.

16. Bobb A, Gleason K, Husch M, Feinglass J, Yarnold PR, Noskin GA. The epidemiology of prescribing errors: The potential impact of computerized prescriber order entry. Arch Intern Med 2004;164(7):785-92.

17. Eppenga WL, Derijks HJ, Conemans JM, Hermens WA, Wensing M, De Smet PA. Comparison of a basic and an advanced pharmacotherapyrelated clinical decision support system in a hospital care setting in the Netherlands. J Am Med Inform Assoc 2012;19(1):66-71.

18. Scheepers-Hoeks AM. Alerts Methods as Success Factors: Influencing Effectiveness of a Clinical Decision Support System in Clinical Practice. [Thesis]. Netherlands: University of Technology Eindhoven; 2014. 